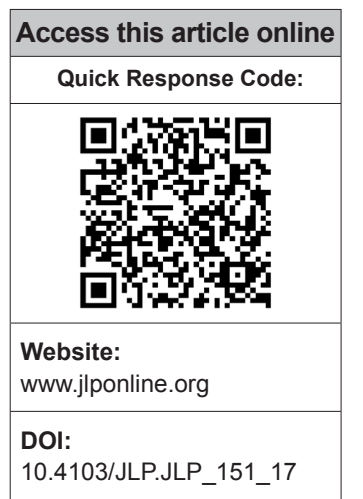

DOI:

10.4103/JLP.JLP_151_17

\section{Comparisons of metabolite profile from paired serum and ethylenediaminetetraacetic acid-plasma samples using dry chemistry technology: An emergency department perspective}

\author{
Lokesh Kumar Sharma, Deep Dutta', Neera Sharma, Bhaskar Thakur ${ }^{2}$
}

\section{Abstract:}

BACKGROUND: No data is available evaluating the difference in serum versus plasma sample assay of commonly tested parameters in the emergency department, where the sample processing time can be significantly reduced if plasma is used for analysis instead of conventionally used serum. Hence, this study aimed to evaluate the differences in serum versus plasma sample estimation of commonly evaluated biochemical parameters using dry chemistry technology.

MATERIALS AND METHODS: Paired blood samples were collected from a single venipuncture of 405 patients admitted to the emergency department. Dry chemistry autoanalyzer (Vitros-350, Ortho Clinical Diagnostics) was used to process all the samples.

RESULTS: Data from 401 patients were analyzed. Percentage differences between serum versus plasma samples for all analytes ranged from $0.0 \%$ to $57.44 \%$ and were $< \pm 4 \%$ for a majority of parameters, except uric acid $(-6.25 \%)$, albumin $(+11.90 \%)$, chloride $(-5.05 \%)$, phosphorus $(-6.06 \%)$, creatine phosphokinase (CPK) total $(-57.44 \%)$, amylase $(-37.53 \%)$, lipase $(-42.74 \%)$, lactate dehydrogenase (LDH) $(-8.53 \%)$, and C-reactive protein (-7.44\%). For albumin, CPK total, amylase, and lipase, the difference between serum and plasma samples was more than the accepted upper range recommended by College of American Pathologists.

Departments of

Biochemistry and

${ }^{1}$ Endocrinology,

Post Graduate Institute of Medical Education and

Research and

Dr. Ram Manohar Lohia

Hospital, New Delhi,

${ }^{2}$ Department of

Biostatistics, Kalinga

Institute of Medical

Sciences, Bhubaneswar,

Odisha, India

Address for correspondence:

Prof. Deep Dutta,

Department of

Endocrinology, Post

Graduate Institute of

Medical Education and

Research and

Dr. Ram Manohar Lohia Hospital, New Delhi, India.

E-mail: deepdutta2000@ yahoo.com

Submission: 04-10-2017

Accepted: 14-01-2018

CONCLUSION: Glucose, urea, creatinine, bilirubin, total protein, serum glutamate-pyruvate transaminase, total cholesterol, high-density lipoprotein cholesterol, triglycerides, sodium, and CPK-mb can be reliably assayed from either serum or plasma samples in emergency/routine practice. CPK total, amylase, and lipase should always be assayed from serum and not plasma due to significant variations. Uric acid, chloride, phosphorous, and LDH only in emergency situations should be assayed from plasma. For routine assays, serum should be preferred.

Key words:

Dry chemistry technology, emergency department, plasma, serum

\section{Introduction}

C onventionally, serum is the most commonly used matrix for biochemical assays in the clinical laboratory. The principle advantage of using serum as

This is an open access journal, and articles are distributed under the terms of the Creative Commons Attribution-NonCommercial-ShareAlike 4.0 License, which allows others to remix, tweak, and build upon the work non-commercially, as long as appropriate credit is given and the new creations are licensed under the identical terms.

For reprints contact: reprints@medknow.com matrix is that reference ranges are readily available for all the different biochemical systems and platforms used for assay. However, serum separation requires at least 20-30 min standby time, which may be further increased in cooler temperatures.

How to cite this article: Sharma LK, Dutta D, Sharma N, Thakur B. Comparisons of metabolite profile from paired serum and ethylenediaminetetraacetic acid-plasma samples using dry chemistry technology: An emergency department perspective. J Lab Physicians 2018;10:346-50. 
This leads to a significant increase in the turnaround time for an investigation (time taken from blood sample collection, processing, analysis, and the final report reaching the clinician). Although it may not be important for elective investigations, this increased turnover time can lead to a significant delay in the clinical decision-making by the treating doctor in the Emergency Department, which can have an adverse impact on patient morbidity and mortality. As a consensus, $<60 \mathrm{~min}$ is considered to be an ideal acceptable turnover time for laboratory investigations (sample registration to result reporting) for common laboratory tests. ${ }^{[1]}$ Advantages of ethylenediaminetetraacetic acid (EDTA) anticoagulated plasma for biochemical analysis include reduced time for sample processing (as no time lost for serum generation). Also for certain investigations such as hemogram, only EDTA anticoagulated blood sample is used. If all the biochemical investigations can be done from EDTA anticoagulated plasma, it will reduce the need for collection of blood samples in different vials at the time of sample collection, thus reducing the volume of blood sample needed for biochemical investigations. Sometimes, there may be a restriction to the volume of blood sample that can be collected from a patient based on the clinical status of the patient.

However, there are a few reports to suggest that significant difference may be observed when the same biochemical parameter is measured in serum and plasma samples based on the platform used for analysis. ${ }^{[2,3]}$ Incorrect reporting may lead to diagnosis of nonexistent disorders (labomas), which can lead to significant patient morbidity and mortality. ${ }^{[4,5]}$

Dry chemistry technology is increasingly being used in large laboratory setups. Advantages of dry chemistry technology include the ability to process a large number of samples in a relatively shorter duration of time along with a good accuracy of testing. However, till date, no data is available evaluating the difference in serum versus plasma sample assay of commonly tested biochemical parameters in clinical practice. Hence, the aim of this study was to evaluate the differences in serum versus plasma sample estimation of commonly evaluated biochemical parameters using dry chemistry technology in a large tertiary care center of India.

\section{Materials and Methods}

Paired blood samples were collected from a single venipuncture of 405 patients admitted to the emergency department of a large tertiary care center in India. Blood samples were collected in plain as well as K2-EDTA vacutainer tubes (Becton Dickinson). K2-EDTA plasma and serum were separated by centrifugation using standard guidelines and processed for estimation of glucose, urea, creatinine, uric acid, total bilirubin (TBil), direct bilirubin (DBil), aspartate transaminase, alanine transaminase, total protein, albumin, total cholesterol (TC), direct high-density lipoprotein (HDL), triglycerides (TGs), sodium, chloride, phosphorus, creatine phosphokinase total, CPK-MB (CPKmb), lactate dehydrogenase (LDH), amylase, lipase, and C-reactive protein (CRP). Dry chemistry autoanalyzer (Vitros-350, Ortho Clinical Diagnostics) was used to process all the samples. The Institute Ethics Committee approved the study protocol. The study protocol was explained and informed written consent was taken from all patients before sample collection for this study.

\section{Dry chemistry technology}

The main principle of dry chemistry is based on the reflectance spectrophotometry in which the reflectance of the materials is measured to give a reference standard for the comparison of the color of the different samples. In dry chemistry technology, the slides are dry, multilayered analytical elements coated onto polyester support. A small amount of patient sample is deposited onto slide and evenly distributed to all the layers. The spreading layer consists of appropriate substance and other components needed for the chemical reaction to occur. The analyte in the sample catalyzes the reaction sequence to yield products which absorb light at wavelengths in various regions (340-680 $\mathrm{nm}$ ), diffuses into underlying layer, and is monitored by reflectance spectrophotometry. The tests performed were colorimetric, enzymatic endpoint, two-point or multipoint rate, or potentiometric analysis depending on the analysate. The CRP was measured by immunoturbidimetric assay. Advantages of DCT include the extremely small amount of sample needed for analysis $(10 \mu \mathrm{L})$, accuracy, and high volumes of patient samples being analyzed over a shorter period.

The methods used for analysis, the assay range, and intra- and inter-assay coefficient of variation for each of the biochemical parameter evaluated have been elaborated in Table 1.

\section{Statistical analysis}

Normality of the distribution of variables was assessed using the Kolmogrov-Smirnov test. Parametric and nonparametric tests were used for analysis for normally distributed and skewed variables, respectively. Pearson's $(r)$ or Spearman's $(\sigma)$ correlation coefficient was calculated for normally distributed and skewed variables, respectively. SPSS version 20 (Chicago, IL, USA) was used for data analysis.

\section{Results}

For the collected samples from 405 patients, data from 401 patients were analyzed. Four pairs of samples 
Table 1: Details of the methods used for biochemical analysis of different evaluated parameters in this study with their assay range, minimal detectable concentration, and their inter- and intra-assay coefficient of variations

\begin{tabular}{|c|c|c|c|c|c|c|c|c|}
\hline Parameter & Method & MDC I & L1-concentration & Intra-assay & nter-assay & & Intra-assay & Inter-assay \\
\hline & & & & CV (\%) & CV (\%) & concentration & CV (\%) & CV (\%) \\
\hline Glucose $(\mathrm{mg} / \mathrm{dl})$ & GOD-POD & 20 & 83 & 0.48 & 1.5 & 292 & 0.38 & 1.2 \\
\hline Urea $(\mathrm{mg} / \mathrm{dl})$ & Urease & 4.29 & 42.9 & 1 & 1.5 & 107 & 0.8 & 1.6 \\
\hline Creatinine (mg/dl) & Creatinine amidohydrolase & 0.05 & 0.41 & 2.93 & 4.8 & 13.58 & 0.72 & 1 \\
\hline Uric acid (mg/dl) & Uricase & 0.5 & 4.4 & 0.91 & 1.7 & 10.3 & 0.68 & 1.1 \\
\hline Bilirubin (mg/dl) & Dyphylline & 0.1 & 1.2 & 1.7 & 2.4 & 15.2 & 0.66 & 1.7 \\
\hline SGPT (U/L) & LDH-PLP & $3 U / L$ & 44 & 3.63 & 6.6 & 187 & 1.02 & 1.9 \\
\hline Total protein (g/dl) & Biuret & $2 \mathrm{~g} / \mathrm{dL}$ & 4 & 1 & 2 & 7.4 & 0.81 & 1.2 \\
\hline Albumin $(g / d l)$ & $\mathrm{BCG}$ & 1 & 2.4 & 0.83 & 1.7 & 4.6 & 0.65 & 0.9 \\
\hline Total cholesterol (mg/dl) & Cholesterol ester hydrolase & 50 & 147 & 0.68 & 1.8 & 259 & 0.7 & 1.5 \\
\hline $\mathrm{dHDL}-\mathrm{C}(\mathrm{mg} / \mathrm{dl})$ & $\mathrm{PTA} / \mathrm{MgCl}_{2}$ & 5 & 37.5 & 2.7 & 2.9 & 65.7 & 2.3 & 3 \\
\hline Triglyceride (mg/dl) & Glycerol kinase & 10 & 104 & 0.96 & 1.1 & 464 & 1.12 & 1.4 \\
\hline Sodium (mmol/L) & Direct potentiometry & 75 & 119 & 0.42 & 0.6 & 152 & 0.4 & 0.6 \\
\hline Chloride (mmol/L) & Direct potentiometry & 50 & 84 & 0.5 & 0.7 & 122 & 0.41 & 0.6 \\
\hline Phosphorus (mg/dl) & Ammonium molybdate & 0.5 & 3.6 & 0.83 & 2.4 & 7.1 & 0.7 & 1.5 \\
\hline CPK total (U/L) & $\mathrm{NAC}-\mathrm{Mg}+2$ & 20 & 145 & 1.4 & 3.7 & 769 & 1.65 & 3.2 \\
\hline CPK-MB & Anti CK-M, NAC-Mg+2 & 2.7 & 22 & 2.3 & 3.4 & 47 & 1.3 & 1.9 \\
\hline $\mathrm{LDH}$ & Pyruvate-NADH & 100 & 441 & 2.6 & 3.3 & 1455 & 1.5 & 2.1 \\
\hline Amylase & Amylopectin & 30 & 74 & 4.3 & 5.2 & 313 & 2.1 & 2.5 \\
\hline Lipase & Colipase & 10 & 159 & 1.7 & 2.1 & 674 & 0.9 & 1.4 \\
\hline C-reactive protein & Immuno-rate assay & 5 & 21 & 4.8 & 8.4 & 69 & 3.5 & 3.8 \\
\hline
\end{tabular}

$\mathrm{MDC}=$ Minimal detectable concentration, CV = Coefficient of variation, SGOT = Serum glutamic oxaloacetic transaminase, SGPT = Serum glutamate-pyruvate transaminase, dHDL-C = Direct high-density lipoprotein cholesterol, $\mathrm{CPK}=$ Creatine phosphokinase, $\mathrm{LDH}=\mathrm{Lactate}$ dehydrogenase, $\mathrm{CRP}=\mathrm{C}$-reactive protein, GOD = Glucose oxidase, POD = Peroxidase, PLP = Pyridoxal phosphate, NAC = N-acetyl cysteine, NADH = Nicotinamide adenine dinucleotide, $\mathrm{CPK}-\mathrm{MB}=$ Creatine phosphokinase-MB, CK-M = Creatine kinase-M

were excluded from the analysis due to the presence of significant hemolysis. Percentage differences between serum versus anticoagulated plasma samples for all analytes ranged from $0.0 \%$ to $57.44 \%$ and were $< \pm 4 \%$ for a majority of parameters, except for uric acid $(-6.25 \%)$, albumin $(+11.90 \%)$, chloride $(-5.05 \%)$, phosphorus $(-6.06 \%)$, CPK total $(-57.44 \%)$, amylase $(-37.53 \%)$, lipase $(-42.74 \%), \mathrm{LDH}(-8.53 \%)$, and CRP (-7.44\%) [Table 2]. A significant positive correlation was observed between serum and plasma values of all the biochemical parameters evaluated.

When we compare the results of our study with the maximum allowable error from College of American Pathologists (CAP) Chemistry Survey reports, ${ }^{[3]}$ we observe that albumin, CPK total, amylase, and lipase - the biochemical parameters where the difference between serum and plasma samples - were more than the accepted upper range recommended by CAP.

Correlation between serum and plasma values for all the parameters evaluated in this study was statistically significant $(P<0.001)$, with the correlation coefficient for glucose, urea, creatinine, uric acid, TBil, DBil, indirect bilirubin, serum glutamate-pyruvate transaminase (SGPT), total protein, albumin, TC, HDL-C, TGs, sodium, chloride, phosphorus, CPK total, CPK-MB, $\mathrm{LDH}$, amylase, lipase, and CRP being $0.973,0.979,0.980$, 0.960, 0.927, 0.806, 0.919, 0.996, 0.908, 0.928, 0.952, 0.867,
$0.739,0.350,0.655,0.404,0.308,0.263,0.911,0.885,0.994$, and 0.996 , respectively.

\section{Discussion}

Significant data are available in literature highlighting the impact of the nature of blood sample used for a biochemical analysis on the final result. A study published in 1990 highlighted that when finger prick blood was used for serum cholesterol measurement, it resulted in increased measurement of serum cholesterol with an average positive bias $+2.4 \%$ as compared to serum levels, resulting in a substantial larger number of patients being labeled with hypercholesterolemia. ${ }^{[6]}$ In a small study of 24 volunteers in 1994 revealed significant variance in serum and plasma levels of TC, TGs, and HDL-C on different assay platforms. ${ }^{[7]}$

Studies have demonstrated that the nature of the blood sample collected (serum vs. plasma) may have an impact even on epigenetic studies, due to their potential interference with biological processes such as DNA methylation during the sample collection. ${ }^{[8]}$ Matrix metalloproteinases (MMPs) have been used as useful diagnostic or prognostic markers in different malignancies. Studies have shown that there is a significant difference in MMP levels depending on when plasma or serum samples were used for analysis, and plasma samples, in general, are preferred for 
Table 2: Difference between serum and plasma values of selected biochemical tests using dry chemistry autoanalyzer (Vitros-350, Ortho Clinical Diagnostics) from blood samples obtained from emergency department $(n=401)$

\begin{tabular}{|c|c|c|c|c|c|c|}
\hline \multirow{2}{*}{$\begin{array}{l}\text { Parameter (units) } \\
\text { (normal range) }\end{array}$} & \multicolumn{2}{|c|}{ Specimen analyzed } & \multirow{2}{*}{$\begin{array}{c}\text { Absolute } \\
\text { difference (P-S) }\end{array}$} & \multirow{2}{*}{$\begin{array}{c}\text { Percentage } \\
\text { difference (median) }\end{array}$} & \multirow[t]{2}{*}{ MAE (\%) } & \multirow[t]{2}{*}{$P^{\#}$} \\
\hline & Serum (S) & Plasma (P) & & & & \\
\hline Glucose (mg/dl) (70-100) & $107.8 \pm 40.29$ & $110.24 \pm 41.46$ & $3(1-4)$ & 2.43 & 10 & $<0.001$ \\
\hline Urea (mg/dl) (15-45) & $24.17 \pm 14.35$ & $23.24 \pm 14.62$ & $-1.0(-1.1-0.0)$ & -3.74 & 9 & $<0.001$ \\
\hline Creatinine (mg/dl) (0.6-1.2) & $0.80 \pm 0.55$ & $0.78 \pm 0.55$ & $0.00(-0.1-0.0)$ & 0.00 & 15 & $<0.001$ \\
\hline Uric acid (mg/dl) (2.5-6.5) & $5.31 \pm 1.33$ & $4.97 \pm 1.25$ & $-0.3(-0.5--0.2)$ & -6.25 & 17 & $<0.001$ \\
\hline Total bilirubin (mg/dl) $(0.2-1.3)$ & $0.76 \pm 0.35$ & $0.72 \pm 0.34$ & $0.0(-0.1-0.0)$ & 0.00 & 20 & $<0.001$ \\
\hline Direct bilirubin (mg/dl) & $0.26 \pm 0.16$ & $0.26 \pm 0.15$ & $0.0(0.0-0.1)$ & 0.00 & 20 & 0.582 \\
\hline Indirect bilirubin (mg/dl) & $0.49 \pm 0.30$ & $0.45 \pm 0.29$ & $0.0(-0.1-0.0)$ & 0.00 & 20 & $<0.001$ \\
\hline SGPT (U/L) $(10-50)^{*}$ & $36(27-49)$ & $37(29-51)$ & $1.0(-1.0-3.0)$ & 2.63 & 20 & 0.043 \\
\hline Total protein $(\mathrm{mg} / \mathrm{dl})(6.3-8.2)$ & $7.48 \pm 0.59$ & $7.61 \pm 0.59$ & $0.10(0.0-0.3)$ & 1.42 & 10 & $<0.001$ \\
\hline Albumin (mg/dl) (3.5-5.0) & $4.32 \pm 0.47$ & $4.82 \pm 0.50$ & $0.5(0.4-0.6)$ & 11.90 & 10 & $<0.001$ \\
\hline Total cholesterol (mg/dl) $(<200)$ & $168.86 \pm 39.93$ & $165.27 \pm 39.93$ & $-3.0(-7.0-1.0)$ & -1.99 & 10 & $<0.001$ \\
\hline $\mathrm{HDL}-\mathrm{C}(\mathrm{mg} / \mathrm{dl})(>40)$ & $44.62 \pm 12.52$ & $45.38 \pm 14.46$ & $1.0(-1.0-2.0)$ & 1.81 & 30 & $<0.001$ \\
\hline Triglycerides $(\mathrm{mg} / \mathrm{dl})(<150)^{*}$ & $131(91-184)$ & $127(86-179)$ & $-3.0(-6.0-1.0)$ & -2.75 & 25 & 0.043 \\
\hline Sodium (mmol/L) (137-145) & $141.89 \pm 7.73$ & $139.94 \pm 3.83$ & $-2.0(-4.0--1.0)$ & -1.41 & 2.9 & $<0.001$ \\
\hline Chloride (mmol/L) (95-110) & $104.11 \pm 4.54$ & $98.83 \pm 7.66$ & $-5.0(-7.0--4.0)$ & -5.05 & 5 & $<0.001$ \\
\hline Phosphorus (mg/dl) (2.5-4.5) & $3.87 \pm 1.62$ & $3.55 \pm 0.67$ & $-0.2(-0.3--0.2)$ & -6.06 & 10 & $<0.001$ \\
\hline CPK total (U/L) $(50-170)^{\star}$ & $83(55-117)$ & $25(20-49)$ & $-39(-76--17)$ & -57.44 & 30 & $<0.001$ \\
\hline CPK-mb (U/L) (10-50)* & $13(9-19)$ & $13(9.0-18)$ & $0.0(-2.0-2.0)$ & 0.00 & 30 & 0.368 \\
\hline LDH (U/L) (200-600) & $499(426-584)$ & $442(394-501)$ & $-43(-77--20)$ & -8.53 & 20 & $<0.001$ \\
\hline Amylase (U/L) (30-110) & $70(55-87)$ & $42(34-52)$ & $-27(-39.5--17)$ & -37.53 & 30 & $<0.001$ \\
\hline Lipase (U/L) (23-300) & $101(67-161)$ & $59(38-93)$ & $-44(-69--28)$ & -42.74 & 30 & $<0.001$ \\
\hline CRP $(\mathrm{mg} / \mathrm{L})(<1.0)$ & $0.4(0.1-0.7)$ & $0.33(0.10-0.6)$ & $-0.05(-0.11-0.0)$ & -7.44 & 14.3 & $<0.001$ \\
\hline
\end{tabular}

${ }^{*}$ All nonnormally distributed variables expressed as median $\left(25^{\text {th }}-75^{\text {th }}\right.$ percentile $), P<0.05$ considered statistically significant, ${ }^{*}$ Measure of difference between the absolute serum and plasma values, percentage difference $=[(\mathrm{P}-\mathrm{S}) / \mathrm{S}] \times 100$. Normally distributed variables expressed as mean $\pm \mathrm{SD}$. MAE $=\mathrm{Maximum}$ allowable error from CAP chemistry survey reports, SGOT = Serum glutamic oxaloacetic transaminase, SGPT = Serum glutamate-pyruvate transaminase, HDL-C = High-density lipoprotein cholesterol, CPK = Creatine phosphokinase, $\mathrm{LDH}=$ Lactate dehydrogenase, $\mathrm{CRP}=\mathrm{C}$-reactive protein, $\mathrm{SD}=\mathrm{Standard}$ deviation, $\mathrm{CAP}=$ College of American Pathologists, CPK-mb = Creatine phosphokinase-mb, CK-M = Creatine kinase-M

analysis due to the higher levels reported from plasma samples. ${ }^{[9]}$

This is the largest ever study reported, comparing the serum and plasma levels of commonly used anaylates in the biochemistry department. The study highlights that for most of the biochemical parameters which are evaluated in the emergency department, the testing can interchangeably be done on serum or plasma samples without any significant difference in the laboratory results. These parameters, which can be freely assayed on serum or plasma samples using dry chemistry technology, include glucose, urea, creatinine, bilirubin, total protein, SGPT, total cholesterol, HDL-C, TGs, sodium, and CPK-mb. This is because the percentage difference between serum and plasma samples was consistently $< \pm 4 \%$. Hence, even in routine clinical practice, either serum or plasma can interchangeably be used for analysis, without any impact on the outcome result.

It must be highlighted that the parameters which should be assayed preferably on serum samples, and not plasma samples due to significant associated differences, include CPK total, amylase, and lipase, as the percentage differences for this parameters were more than the cutoffs provided by CAP. For parameters such as uric acid, chloride, phosphorous, and LDH, although the difference between serum and plasma samples was more than $\pm 4 \%$, it was less than the cutoffs provided by CAP. Hence, only in real emergency scenarios should these parameters be evaluated from plasma samples. In routine clinical practice, we should continue to evaluate these parameters from serum samples preferably.

\section{Conclusion}

It may be said that said that serum versus plasma samples should not be interchangeably be used in routine clinical practice unless specific data for the same are available depending on the assay platform used for analysis.

\section{Financial support and sponsorship}

Nil.

\section{Conflicts of interest}

There are no conflicts of interest.

\section{References}

1. Hawkins RC. Laboratory turnaround time. Clin Biochem Rev 2007;28:179-94. 
2. Ladenson JH, Tsai LM, Michael JM, Kessler G, Joist JH. Serum versus heparinized plasma for eighteen common chemistry tests: Is serum the appropriate specimen? Am J Clin Pathol 1974;62:545-52.

3. Miles RR, Roberts RF, Putnam AR, Roberts WL. Comparison of serum and heparinized plasma samples for measurement of chemistry analytes. Clin Chem 2004;50:1704-6.

4. Dutta D, Chowdhury S. Endocrine labomas. Indian J Endocrinol Metab 2012;16:S275-8.

5. Society for Promotion of Education in Endocrinology and Diabetes (SPEED) Group, Chittawar S, Dutta D, Khandelwal D, Singla R. Neonatal endocrine labomas - Pitfalls and challenges in reporting neonatal hormonal reports. Indian Pediatr 2017;54:757-62.
6. Greenland P, Bowley NL, Meiklejohn B, Doane KL, Sparks CE Blood cholesterol concentration: Fingerstick plasma vs venous serum sampling. Clin Chem 1990;36:628-30.

7. Beheshti I, Wessels LM, Eckfeldt JH. EDTA-plasma vs serum differences in cholesterol, high-density-lipoprotein cholesterol, and triglyceride as measured by several methods. Clin Chem 1994;40:2088-92

8. Quinlivan EP, Crider KS, Zhu JH, Maneval DR, Hao L, Li Z, et al. Hypomethylation of serum blood clot DNA, but not plasma EDTA-blood cell pellet DNA, from Vitamin B12-deficient subjects. PLoS One 2013;8:e65241.

9. Jonsson A, Hjalmarsson C, Falk P, Ivarsson ML. Levels of matrix metalloproteinases differ in plasma and serum - Aspects regarding analysis of biological markers in cancer. Br J Cancer 2016;115:703-6. 